\title{
Performance Evaluation of an Optical Burst Switched Core Node With Generalized Arrivals and Partial Wavelength Conversion Capability
}

\author{
Mohamed H. Morsy-Osman, Mohammed Y. S. Sowailem, Ahmed I. Abd El-Rahman, and \\ Hossam M. H. Shalaby
}

\begin{abstract}
Many of the burst assembly algorithms employed in optical burst switching (OBS) networks preserve the IP traffic self-similarity property in the burst traffic. We introduce a mathematical model for performance evaluation of an OBS core node employing either no, a partial or a full wavelength conversion strategy. The model assumes long-range dependent (LRD) traffic arrivals to the OBS intermediate node whose inter-arrival times are accurately modeled by a Pareto distribution, whereas exponential holding times are assumed. In our proposed model, each output port in the node is modeled as a $G I / M / w / w$ queue with partial server accessibility. An imbedded Markov chain approach is used to derive the limiting state probability distribution for the number of bursts currently served by an output port as seen by arriving bursts. Next, the average burst loss probability is evaluated from steady-state occupancy probabilities. In addition, the results of our mathematical model are validated via simulation. Furthermore, the results of the model are compared with those when assuming short-range dependent Poisson arrivals. Comparison shows that traditional Poisson traffic models yield over-optimistic performance measures compared to the LRD Pareto traffic models, especially for light traffic scenarios. Furthermore, we study the impact of varying different traffic parameters, such as the average arrival rate and the Hurst parameter, on the burst loss probability. Finally, the impact of varying the wavelength conversion capability on the burst loss probability is studied, where we compare two strategies for contention resolution: adding new channels (wavelengths) or adding wavelength converters, while taking the cost into consideration.
\end{abstract}

Index Terms-Optical burst switched (OBS) networks; Pareto distribution; Queueing theory.

Manuscript received June 12, 2012; revised October 4, 2012; accepted October 11, 2012; published November 20, 2012 (Doc. ID 170034).

Mohamed H. Morsy-Osman is with the Electrical and Computer Engineering Department, Faculty of Engineering, McGill University, Montreal, QC H3A 2A7, Canada, and he is also with the Electrical Engineering Department, Faculty of Engineering, Alexandria University, Alexandria 21544, Egypt.

Mohammed Y. S. Sowailem is with the Electrical and Computer Engineering Department, Faculty of Engineering, McGill University, Montreal, QC H3A 2A7, Canada.

Ahmed I. Abd El-Rahman (e-mail: ahmedibrahim766@hotmail.com) is with the Electrical Engineering Department, Faculty of Engineering, Alexandria University, Alexandria 21544, Egypt.

Hossam M. H. Shalaby is with the School of Electronics, Communications, and Computer Engineering, Egypt-Japan University of Science and Technology (E-JUST), New Borg El Arab City, Alexandria 21934, Egypt, and is on leave from the Electrical Engineering Department, Faculty of Engineering, Alexandria University, Alexandria 21544, Egypt.

Digital Object Identifier 10.1364/JOCN.4.000997

\section{INTRODUCTION}

$\mathbf{R}$ ecent research work has aimed at adapting communication networks to the huge surge in Internet traffic. This has allowed advanced suites of technologies and protocols, e.g., generalized multiprotocol label switching (GMPLS), to appear as effective solutions that support the extremely rapid growth in Internet traffic. Nevertheless, it remains a questionable issue whether there exists an effective solution to the all-optical processing and buffering problem. In that regard, optical burst switching (OBS) has been developed as a switching paradigm that can support traffic streams introduced by upper layer protocols which are bursty in nature. The idea of OBS, first proposed by researchers in [1,2], has emerged to make all-optical networks (AONs) realizable by combining the best of both optical circuit switching (OCS) and optical packet switching (OPS). In particular, OBS avoids optical buffering and effectively addresses the all-optical data processing problem by separating the header from its payload and performing per burst switching operations. In OBS, a data burst (DB) carries the payload that is composed of a group of aggregated IP packets having the same destination and class, whereas the burst header referred to as the control packet (CP) bears information required for routing operations. In this way, the $\mathrm{CP}$ encompasses all electronic processing while the DB can travel through the whole network without being optically buffered or electronically processed. The variability in DB length from as short as a packet to as long as a session places OBS as an intermediate solution between OCS and OPS that is more matched to bursty traffic over the Internet.

The OBS network (OBSN) consists of three components: ingress, core and egress nodes [3]. The ingress node is the node at which the aggregation process of packets occurs to form a DB that enters the core network [4]. Core nodes route DBs according to their CPs that have already been received and processed prior to the arrival of their corresponding DBs, which is the benefit of introducing an offset time between the $\mathrm{CP}$ and the $\mathrm{DB}$ at the ingress node to ensure a bufferless core. The egress node is the destination node where the DB is disassembled back into original packets, each of which is directed to its own destination [4].

One-way reservation protocols such as just-enough-time (JET) [2] and just-in-time (JIT) [5] are commonly used for 
signaling in OBS. Such protocols suffer from unacceptable burst dropping probabilities due to the probable contention between two CPs while reserving resources for their ensuing DBs.

Various techniques have been proposed in the literature for contention resolution; conventional contention resolution techniques include fiber delay lines (FDLs) and deflection routing. In [6], Wu et al. suggested an efficient two phase channel scheduler for optical burst switched networks. In principle, the proposed scheduling strategy aims at finding feasible voids on all data channels, then choosing the optimal voids among these voids. Moreover, a configurable void selection approach has been designed to support various scheduling algorithms, which would boost up the network performance by efficiently utilizing network resources. Bonani has presented a performance analysis of OBS mesh networks using wavelength division multiplexing (WDM) and localized functional resources (LFC) [7]. In this topology, the network design implies assigning the same number of wavelengths to all network links and adding contention resolution schemes like wavelength converters and deflection routing capability on nodes or links expecting higher traffic. The author has shown that the LFC would enhance the performance of metropolitan area networks (MANs) without increasing the number of wavelengths on each link.

Furthermore, in order to reduce the burst loss probability with fixed per-hop delay, Ichikawa and Kamakura have determined a forward-resource-reservation- (FRR-) based scheduler buffer size [8]. They have developed an edge node model to analyze the suggested scheme. In addition, they have been able to show the existence of an optimal buffer size that depends on both the number of aggregated packets in a burst and the packet arrival rate. Moreover, they have shown that FRR-based schemes achieve a much lower minimum packet loss probability than non-FRR-based OBS systems.

On the other hand, Abd El-Rahman et al. proposed a different technique based on CP buffering [9]. Other contention techniques are based on employing wavelength or code converters in the core node resources [10,11]. Either FWC (full wavelength conversion) or PWC (partial wavelength conversion) may be adopted. In FWC, a DB arriving at a channel can be switched onto any other idle channel on its destination output port. FWC significantly reduces burst loss probabilities compared to no wavelength conversion (NWC). However, the implementation of all-optical FWC is very costly. Thus, PWC, in which only a limited number of the available wavelengths are convertible, is proposed when there is a limited number of tunable wavelength converters (TWCs), and consequently some DBs may be dropped when all converters are busy despite there being free channels on the output port. Motivated by the cost-effectiveness of PWC, our paper studies an OBS core node employing PWC with full-range TWCs implemented in a dedicated-per-input-line (DPIL) strategy similar to the one assumed in [12].

In [13], Rosberg et al. presented a scalable framework for estimating path blocking probabilities in OBS networks where limited wavelength conversion is possible. They built their framework on the classical reduced load approximation to calculate link blocking probabilities from which path blocking probabilities were estimated. Moreover, the limitation in wavelength conversion came from the limited conversion range. Wavelength converters with a limited conversion range allow an incoming wavelength to be switched only to a small subset of outgoing wavelengths, referred to as the range of conversion. Furthermore, in [14] the blocking time distributions for an OBS node assuming Poisson-arriving bursts with Pareto, Gaussian and exponential burst size distributions were obtained. Such expressions can be used to dimension FDLs needed for contention resolution and to perform end-to-end delay estimation. Meanwhile, the authors in $[15,16]$ were concerned with OBS networks in which the variability of the burst length would result in occupying the same wavelength on two adjacent links simultaneously. Finally, [17] aimed at evaluating the burst loss probability in an OBS network that adopts FDLs, implemented in a share-per-node architecture, as a contention resolution technique. In particular, the paper proposed replacing the conventional Poisson arrivals model by a two moment traffic model. Although not exact, the proposed model represents a better approximation than usual Poisson models.

Turning to the traffic model adopted for DB arrivals, it is clear that the confidence of results obtained from mathematical models built for performance evaluation of realistic networks depends on how close the traffic models are to real traffic streams. For that reason, while building our model, we should model DB arrivals in the most accurate form possible. Statistical analysis of high-resolution traffic measurements from a wide range of running packet networks, such as the Internet, have convincingly shown that actual traffic streams in such networks exhibit the property of self-similarity or long-range dependency (LRD) $[18,19]$. This means that similar statistical patterns may occur over different time scales that can vary by many orders of magnitude (i.e., ranging from milliseconds to hours). Consequently, the behavior of these traffic streams significantly departs from traditional telephone traffic and its related Markov models with short-range dependency (SRD). In particular, the Poisson arrival process and analyses based on the Erlang-B formula would no longer be valid. Alternatively, another probability distribution function rather than the conventional Poisson distribution is needed to model these new statistical properties. The Pareto probability distribution has commonly been used, as a heavy-tailed distribution, as a good fit for such LRD data streams.

Moreover, in [18] simulation results that reveal the effect of burst assembly algorithms, namely, timer-based and hybrid assembly algorithms, on burst traffic statistics are presented. These results show that the self-similarity property is persistent in the assembled traffic in both cases; however, burst traffic exhibits a lower degree of self-similarity. Moreover, Yu et al. presented a detailed analysis of the burst traffic statistics in [4]. In particular, they discussed the LRD property in the assembled burst traffic when the timer-based assembly algorithm is employed and verified their conclusions using simulation. They argued that the LRD property remains unchanged under light traffic conditions as, in this case, the burst processing time is short relative to the burst interleaving time. Hence, the assembly of one burst does not affect the departure time of its following bursts. On the other hand, when the traffic load is medium to heavy, the traffic becomes smoother. Nevertheless, this does not affect the correlation properties at large time scales. Indeed, the use of Poisson 
models remains viable. However, as stated earlier, since the LRD property of burst traffic arises in many occasions, analysis of the performance of an OBS core node with self-similar traffic is strongly relevant. On the other hand, we would like to clarify that our mathematical model is general and hence is not only applicable to OBS networks but can also be used to study a core node of any optical network (including a GMPLS controlled optical network) that utilizes the dedicated-per-input-line (DPIL) scheme for deploying the TWCs. This, of course, requires some measurements or expectations for the traffic of the optical network under test assuming that the length of the data unit used in such a network is exponentially distributed.

Our earlier work in [20] presented a mathematical model that evaluates upper and lower bounds for the burst loss probability of DBs at an OBS core node employing either NWC or FWC, while assuming LRD Pareto distributed inter-arrival times. Improving our work in [20], the newly proposed model in this paper aims to evaluate the performance of an OBS core node in the general PWC case while assuming an arbitrary distribution for the inter-arrival times and exponential service times. Our derived model in this paper is applied to the LRD Pareto distributed inter-arrival times. Intuitively, the model results for the $\mathrm{PWC}$ case prove to be backward compatible with those in special NWC and FWC cases. In the proposed model, each output port in the node is modeled as a $G I / M / w / w$ queue with partial server accessibility, i.e., the queue is assumed to have general independent arrivals, Markovian service times, $w$ servers and no waiting room. Moreover, PWC is modeled by restricting the accessibility of servers by an arriving DB depending on whether its wavelength is a convertible one or not. As this queue does not have a well-known solution in the queueing literature, an imbedded Markov chain approach is used to derive the limiting occupancy probability distribution for the number of customers (DBs) currently served by an output port at the imbedded arrival epochs. Next, the average burst loss probability is obtained from steady-state occupancy probabilities. It is noteworthy that our model applies for any inter-arrival time distribution as long as the independence assumption is preserved.

It should be pointed out that the novelty of the studied problem implies studying the performance of a single core node in isolation from the whole network to simplify the analysis, which is common in the literature [10,13,21-23]. Indeed, the performance of an OBS network is affected by many other factors such as different offset times, the scheduling algorithm used and the burst assembly algorithm adopted. However, study of the performance of a single OBS core node still gives insight into how the node will perform with the parameters assumed in a networking scenario. Also, study of the performance of a single core node allows the building of more complicated mathematical models that assume traffic models (e.g., arrival and service processes) closer to reality.

Finally, the results of our model are first justified by simulation and then used to compare the burst loss probability in two cases: Pareto LRD and Poisson SRD DB arrivals. The comparison shows that conventional Poisson traffic models give lower estimates for the burst loss probability when compared to Pareto traffic models, especially in case of light traffic scenarios, in all NWC, PWC and FWC cases. Furthermore, the effect of varying traffic parameters such as average arrival rate and Hurst parameter (measure of the degree of self-similarity) on the burst loss probability is studied. Finally, the impact of varying the wavelength conversion capability on the burst loss probability is studied, where we compare two strategies for contention resolution: increasing the number of channels or adding extra wavelength converters, while taking cost into consideration.

The remainder of this paper is organized as follows. In Section II, we present the assumptions upon which our model equations are derived in Section III. Section IV is devoted to presenting simulation work, numerical results and discussion of results. Finally, we conclude in Section V.

\section{Proposed Model Assumptions}

This section presents detailed assumptions made about the resources in the core node under study. Specifically, we are going to build our model upon the following set of assumptions:

- The destination output port for an incoming DB to the OBS core node is uniformly distributed among all available output ports. Thus, it is sufficient to model the behavior of a single output port instead of considering all output ports of the node.

- Each OBS core node considered in our model has the following resources [11]:

i. A number $w$ of wavelengths available to serve the incoming DBs. For an incoming DB, all $w$ wavelengths are equally likely.

ii. No FDLs, i.e., no buffering capabilities for contention resolution in the OBS nodes.

iii. A number of wavelength converters, each of which can convert the wavelength of the incoming DB to any other free wavelength from the set of the available $w$ channels whenever a contention occurs. Typically, the set of available wavelengths is denoted by $\Lambda \stackrel{\text { def }}{=}\left\{\lambda_{1}, \lambda_{2}, \ldots, \lambda_{w}\right\}$, while the node has $u$ wavelength converters, where $u \in$ $\{0,1,2, \ldots, w\}$. This means that only $u$ wavelengths of $\Lambda$ can be converted to any other wavelength in $\Lambda$, while the remaining $w-u$ wavelengths are nonconvertible. We define the node conversion capability as $\gamma \stackrel{\text { def }}{=} \frac{u}{w}$. Thus, $\gamma \in[0,1]$ is the measure of the differentiation between the wavelength conversion schemes. The FWC case occurs when $\gamma=1$, while the NWC case occurs when $\gamma=0$ and finally the PWC case occurs when $0<$ $\gamma<1$. The practical meaning of these values can be illustrated as in [12], where the conversion capability can be seen as the ratio between the number of deployed TWCs $(u)$ at each port and the number of available wavelengths $(w)$, i.e., $\gamma=\frac{u}{w}$, where $u \in\{0,1,2, \ldots, w\}$. Thus, the relation between the mathematical model and the practical case depends on the conversion capability $(\gamma)$ (or in other words $u$ ).

- Inter-arrival times between incoming DBs to the OBS core node are independent and identically distributed, drawn from a common Pareto distribution with an average $1 / \lambda$ seconds, where $\lambda$ is the average arrival rate in bursts/second. On the other hand, the service times of DBs are independent and identically distributed, drawn from a common 
exponential (Markovian) distribution with a mean $1 / \mu$ seconds, which is equal to the average $\mathrm{DB}$ duration, where $\mu$ is the average service rate. Furthermore, inter-arrival times and service times are assumed to be independent.

Speaking in more detail about arrival and service processes, we denote by $\mathrm{DB}_{n}$ the $n^{\text {th }}$ arriving customer or $\mathrm{DB}$, as shown in Fig. 1. Then, we define their arrival instant at the node as

$$
\tau_{n} \stackrel{\text { def }}{=} \text { arrival time of } \mathrm{DB}_{n}
$$

and further define the inter-arrival time between $\mathrm{DB}_{n-1}$ and $\mathrm{DB}_{n}$ as

$$
\begin{aligned}
T_{a}^{n} \stackrel{\text { def }}{=} \text { inter-arrival time between } \mathrm{DB}_{n-1} \text { and } \mathrm{DB}_{n} \\
\quad=\tau_{n}-\tau_{n-1} .
\end{aligned}
$$

As already stated, we have assumed that all inter-arrival times are identically distributed, commonly drawn from the two-parameter Pareto distribution having the following form for the probability distribution function (pdf):

$$
f_{T_{a}^{n}}(t)=\frac{a b^{a}}{(b+t)^{a+1}}, \quad \text { where } t>0, a>0 \text { and } b>0
$$

which is independent of $n$. In particular, we will be concerned with the limiting random variable $T_{a}$ having the limiting pdf $f_{T_{a}}$ defined as

$$
\begin{gathered}
T_{a} \stackrel{\text { def }}{=} \lim _{n \rightarrow \infty} T_{a}^{n}, \\
f_{T_{a}} \stackrel{\text { def }}{=} \lim _{n \rightarrow \infty} f_{T_{a}^{n}}=f_{T_{a}^{n}} .
\end{gathered}
$$

As required in the following sections, $f_{T_{a}}(t)$ has its Laplace transform pair $F_{T_{a}}(s)$, derived in [24], given by

$$
F_{T_{a}}(s)=a(b s)^{a} e^{b s} \Gamma(-a, b s),
$$

where $\Gamma(a, x)$ is the incomplete gamma function defined as

$$
\Gamma(a, x)=\int_{x}^{\infty} t^{a-1} e^{-t} d t
$$

It can be easily shown that a Pareto random variable has a finite mean and infinite variance for $1<a<2$, with its mean given by

$$
E\left(T_{a}\right)=\frac{1}{\lambda}=\frac{b}{a-1} .
$$

In order to model a self-similar or LRD traffic stream, we are going to assume that inter-arrival times are Pareto distributed with parameter $a$ set in the interval $(1,2)$. This is to make the mean of the inter-arrival times finite while their variance is infinite. The infinite variance syndrome models the LRD property exhibited by DB traffic, as known in the literature. Moreover, the degree of self-similarity of the traffic is measured by the Hurst parameter denoted by $H$, where $H \in(0,1)$. It is proved that when $H \in(0.5,1)$, the process has a non-summable autocorrelation function (ACF), i.e., the process exhibits the

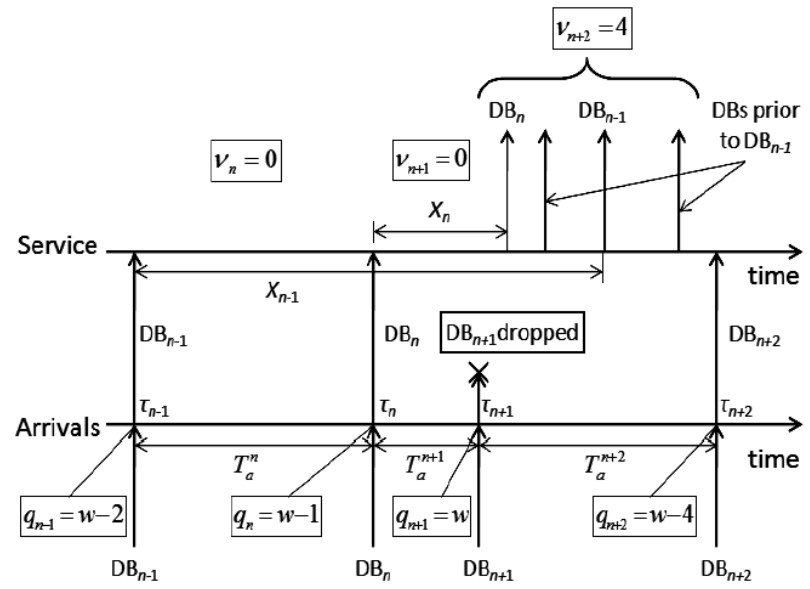

Fig. 1. Arrival scenario to the $G I / M / w / w$ queue showing imbedded Markov points.

same statistical properties for different lag times and hence is self-similar or LRD.

The Hurst parameter $H$ of the arrival process, whose values are drawn from the Pareto distribution, is related to the parameter $a$ of the Pareto pdf as follows:

$$
H=(3-a) / 2 .
$$

It should be noted that, throughout this work, we do not estimate the Hurst parameter of any measured set of traffic since we do not have experimentally measured traffic. Nevertheless, we generate traffic streams that have a well calculated Hurst parameter according to the desired degree of self-similarity for our results using the heavy-tailed Pareto distribution, i.e., the Hurst parameter of the generated traffic is set a priori. Hence, we do not need any of the well-known Hurst parameter estimation techniques used in the literature to estimate the Hurst parameter of a measured traffic stream, e.g., R/S analysis.

Thus, we will just adjust the degree of self-similarity of the generated traffic stream by varying the parameter $a$ in the range $(1,2)$. The closer $H$ is to one, the more self-similarity the traffic possesses. After setting $a$ according to the desired degree of self-similarity, $b$ is chosen according to Eq. (8) depending on the required mean arrival rate of the generated DB traffic.

Similarly, we define the service time of $\mathrm{DB}_{n}$ as

$$
X_{n} \stackrel{\text { def }}{=} \text { service time of } \mathrm{DB}_{n},
$$

and since service times are identically distributed, their common exponential distribution will be independent of $n$ and given by

$$
f_{X_{n}}(x)=\mu e^{-\mu x}, \quad \text { where } x>0 \text {. }
$$

Similarly to the arrivals, we will assume the existence of the following limiting random variable and pdf for service times:

$$
\begin{gathered}
X \stackrel{\text { def }}{=} \lim _{n \rightarrow \infty} X_{n}, \\
f_{X}(x)=\lim _{n \rightarrow \infty} f_{X_{n}}(x)=\mu e^{-\mu x}, \quad \text { where } x>0,
\end{gathered}
$$


with the parameter $\mu$ set according to the desired average DB length.

Upon the aforementioned assumptions, we model each output port in the node as a $G I / M / w / w$ queue with limited server accessibility. For that queue, there are $w$ servers modeling the available $w$ wavelengths per output port. Furthermore, assuming general independent arrivals to the queue allows us to consider the desired Pareto LRD arrivals case; however, the case of Poisson SRD arrivals can also be studied via the derived model equations for the sake of comparison.

Turning to the issue of server accessibility, the key idea is that the $w$ servers are not fully accessible unless the node has FWC capability $(\gamma=1)$; otherwise, for NWC and PWC cases $(\gamma=0$ and $0<\gamma<1)$, free servers are not allowed to be reserved by every incoming $\mathrm{DB}$, i.e., server accessibility is restricted somehow. For instance, in the NWC case, each incoming DB is destined for a specific server that represents the wavelength on which it arrives and it will be blocked if this specific server is busy at its time of arrival, i.e., contention is irresolvable. Moreover, in the PWC case, an incoming DB will be blocked if its own wavelength (server) is busy and its wavelength is nonconvertible, which implies that the free servers (if any) in this case are not accessible by this DB.

Finally, the use of a $G I / M / w / w$ queue that has no waiting room is due to the assumption that no FDLs are deployed in the core node resources. Also, first-in-first-out (FIFO) service order is assumed.

\section{Mathematical Model Equations}

The system under study, a $G I / M / w / w$ queue with partial server accessibility, has its customers arriving with inter-arrival times independently and identically distributed according to a general distribution $f_{T_{a}}(t)$. Accordingly, no assumption is made about the memory of the process, i.e., the arrival process is not memory-less unless it is a Poisson process and consequently $f_{T_{a}}(t)$ is exponential.

If we denote by $N(t)$ the number of customers (or DBs) in the system at any time $t$, then clearly $\{N(t): t \geq 0\}$ is not a continuous-time Markov chain (CTMC) on the state space $S=\{i: i \in W\}$, where $W=\{0,1, \ldots, w\}$, because specifying $N(t)$ is not enough to summarize the complete history of the process prior to $t$. Additionally, we must specify extra information about the elapsed time since the last arrival because of the non-Markovian nature of the arrival process. Thus, if we denote by $X_{0}(t)$ the elapsed time (age) since the last arrival at time $t$, then the vector $\left[N(t), X_{0}(t)\right]$ is a two-dimensional continuous-time Markov process over the continuous state space $S=\{[i, x]: i \in W, 0<x<t\}$, where $W$ is defined as before. Thus, the vector $\left[N(t), X_{0}(t)\right]$ summarizes the complete history of the process prior to $t$. We can use the method of supplementary variables [23] to carry out the analysis by considering the two-dimensional state description $\left[N(t), X_{0}(t)\right]$. Alternatively, we can use the imbedded Markov chain approach to analyze the queue, which is the technique we follow.

In the imbedded Markov chain approach, we study the evolution of $N(t)$ at the arrival epochs [25]. This set of points implicitly carries the information about the elapsed time since the last arrival $X_{0}(t)$. Therefore, let us define:

$$
\begin{aligned}
q_{n} \stackrel{\text { def }}{=} & \text { number of customers in the system immediately } \\
& \text { prior to arrival of } \mathrm{DB}_{n} \\
= & N\left(\tau_{n}^{-}\right) .
\end{aligned}
$$

Clearly, the sequence $\left\{q_{n}\right\}$ forms a discrete-time Markov chain (DTMC), which is referred to as the imbedded chain, over the discrete state space $S=\{i: i \in W\}$, where $W$ is defined as before. Figure 1 shows, as an example, a sequence of arrivals and departures for a $G I / M / w / w$ queue with full server accessibility and defines the arrival instants as the imbedded instants on the time axis at which the imbedded DTMC $\left\{q_{n}\right\}$ can change. In the next two subsections, we study the imbedded DTMC $\left\{q_{n}\right\}$ in the two cases of full and partial server accessibility.

Specifically, we will calculate its transition probabilities from which we will find its steady-state occupancy probability distribution at the imbedded points. Finally, we evaluate our performance measure, namely, the average burst loss probability.

\section{A. GI/M/w/w Queue With Full Server Accessibility}

The $G I / M / w / w$ queue with full server accessibility has a well-known solution given by Takacs [26]; however, we will derive the steady-state occupancy probabilities at the imbedded Markov points (arrival epochs) for this queue from basic principles in order to ease the derivation for the general case where partial server accessibility is considered in the following subsection.

As already stated, the sequence $\left\{q_{n}\right\}$ is a DTMC over the state space $S=\{i: i \in W\}$, where $W=\{0,1, \ldots, w\}$. Now, we start by finding transition probabilities associated with this DTMC, defined as

$$
p_{i j}=\operatorname{Pr}\left\{q_{n+1}=j \mid q_{n}=i\right\} .
$$

Moreover, let us denote by $v_{n+1}$ the number of customers served between the arrivals of $\mathrm{DB}_{n}$ and $\mathrm{DB}_{n+1}$. Then, we see immediately (see Fig. 1) that the following relation should hold:

$$
q_{n+1}=\left\{\begin{array}{l}
q_{n}+1-v_{n+1}, \quad 0 \leq q_{n}<w \\
q_{n}-v_{n+1}, \quad q_{n}=w
\end{array}\right.
$$

Figure 2 shows the state transition probability diagram for the $G I / M / w / w$ queue imbedded DTMC. Clearly, according to the value of $i$, we have two cases for the transition probabilities $p_{i j}$ as follows:

a. Case $i<w$ : In this case, the new arrival is accepted. Obviously, for $j>i+1, p_{i j}=0$. On the other hand, for $0 \leq j \leq$ $i+1$, we have

$$
\begin{aligned}
p_{i j} & =\operatorname{Pr}\left\{i+1-j \text { DBs are served during } T_{a}^{n+1}\right\} \\
& =\operatorname{Pr}\left\{v_{n+1}=i+1-j\right\} \\
& =\int_{0}^{\infty} \operatorname{Pr}\left\{v_{n+1}=i+1-j \mid T_{a}^{n+1}=t\right\} f_{T_{a}}(t) d t .
\end{aligned}
$$




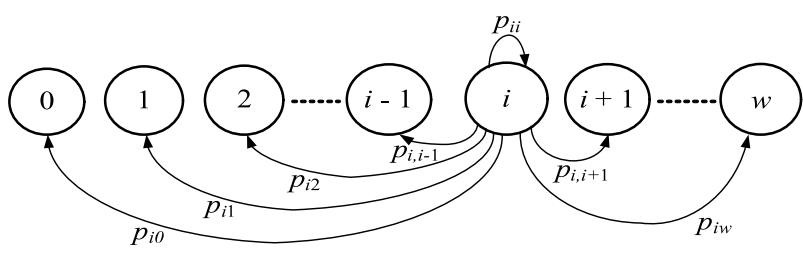

Fig. 2. State transition probability diagram of the imbedded DTMC for $\mathrm{GI} / M / w / w$ showing transitions out of state $i$.

Note that $\operatorname{Pr}\left\{v_{n+1}=i+1-j \mid T_{a}^{n+1}=t\right\}$ represents the probability that exactly $i+1-j$ DBs complete the service out of the $i+1 \mathrm{DBs}$ present during $t$ seconds. Thus, using the binomial distribution and applying the total probability theorem $p_{i j}$ can be written as follows:

$$
p_{i j}=\int_{0}^{\infty}\left(\begin{array}{c}
i+1 \\
i+1-j
\end{array}\right)\left(1-e^{-\mu t}\right)^{i+1-j} e^{-j \mu t} f_{T_{a}}(t) d t
$$

where the binomial coefficient calculates the number of ways in which service completions can occur out of the $i+1 \mathrm{DBs}$ present and can be alternatively written as

$$
\left(\begin{array}{c}
i+1 \\
i+1-j
\end{array}\right)=\left(\begin{array}{c}
i+1 \\
j
\end{array}\right) \text {. }
$$

b. Case $i=w$ : In this case, the new arrival is dropped. Hence, for $0 \leq j \leq w$, we have

$$
\begin{aligned}
p_{i j}=p_{w j} & =\operatorname{Pr}\left\{w-j \text { DBs are served during } T_{a}^{n+1}\right\} \\
& =\int_{0}^{\infty}\left(\begin{array}{c}
w \\
j
\end{array}\right)\left(1-e^{-\mu t}\right)^{w-j} e^{-j \mu t} f_{T_{a}}(t) d t .
\end{aligned}
$$

Now, let us turn to calculating the steady-state occupancy probabilities of the imbedded DTMC. It can be proved that the DTMC $\left\{q_{n}\right\}$ is always ergodic because it is finite, aperiodic and irreducible. Hence, a steady-state occupancy probability distribution $\left\{\pi_{n}: k \in W\right\}$ always exists, where $\pi_{k}$ is defined as

$$
\pi_{k}=\lim _{n \rightarrow \infty} \operatorname{Pr}\left\{q_{n}=k\right\}
$$

Next, to find the steady-state occupancy probabilities $\pi_{k}$, we should solve the following system of linear equations:

$$
\Pi=\Pi P,
$$

where $\Pi=\left[\pi_{0}, \pi_{1}, \ldots, \pi_{w}\right]$ is the occupancy probability vector and $P=\left[p_{i j}\right]$, where $0 \leq i, j \leq w$, is the one-step transition probability matrix of the DTMC.

To get a closed form expression for the steady-state occupancy probabilities $\pi_{k}$, we proceed by calculating the probability generating function (pgf) $\Pi(z)$ of the distribution, defined as

$$
\Pi(z)=\sum_{k=0}^{w} \pi_{k} z^{k}
$$

After substituting for $\pi_{k}$ and $p_{i j}$ from Eqs. (16), (18) and (20) in Eq. (21) and performing some algebraic manipulations, we obtain

$$
\begin{aligned}
\Pi(z)= & \sum_{k=0}^{w} \pi_{k} \int_{0}^{\infty}\left(1-e^{-\mu t}+z e^{-\mu t}\right)^{k+1} f_{T_{a}}(t) d t \\
& +(1-z) \pi_{w} \int_{0}^{\infty} e^{-\mu t}\left(1-e^{-\mu t}+z e^{-\mu t}\right)^{w} f_{T_{a}}(t) d t
\end{aligned}
$$

Then, let us expand the pgf $\Pi(z)$ in a Taylor expansion around $z=1$ as follows:

$$
\Pi(z)=\sum_{r=0}^{w} B_{r}(z-1)^{r},
$$

where the $B_{r}$ are called the binomial moments of the pgf that can be calculated as

$$
B_{r}=\left.\frac{1}{r !} \frac{d^{r} \Pi(z)}{d z^{r}}\right|_{z=1}, \quad r \in W .
$$

As will be seen, it is feasible to obtain a closed form expression for the binomial moments $B_{r}$ of $\Pi(z)$ in terms of the Laplace transform of the inter-arrival time distribution $F_{T_{a}}(s)$, by applying the operator $\left.R_{r} \stackrel{\text { def }}{=} \frac{1}{r !} \frac{d^{r}}{d z^{r}}\right|_{z=1}$ to $\Pi(z)$ given in Eq. (22). Afterwards, we can find the steady-state occupancy probabilities $\pi_{k}$, for $k \in W$, from the binomial moments as follows:

$$
\begin{aligned}
\pi_{k} & =\left.\frac{1}{k !} \frac{d^{k} \Pi(z)}{d z^{k}}\right|_{z=0} \\
& =\left.\frac{1}{k !} \sum_{r=0}^{w} B_{r} \frac{d^{k}}{d z^{k}}\left\{(z-1)^{r}\right\}\right|_{z=0} \\
& =\sum_{r=k}^{k}\left(\begin{array}{l}
r \\
k
\end{array}\right)(-1)^{r-k} B_{r} .
\end{aligned}
$$

Then, the last part is to find the binomial moments $B_{r}$ of $\Pi(z)$ by applying the operator $R_{r}$ to both sides of Eq. (22), which results in the following first order difference equation:

$$
B_{r}=\alpha_{r}\left[B_{r}+B_{r-1}\right]-\alpha_{r}\left(\begin{array}{c}
w \\
r-1
\end{array}\right) B_{w}
$$

where

$$
\alpha_{r}=F_{T_{a}}(r \mu)=\int_{0}^{\infty} f_{T_{a}}(t) e^{-r \mu t} d t .
$$

Noting that $B_{0}=1$, the above difference equation can be solved recursively until $B_{r}$ is obtained as follows:

$$
B_{r}=C_{r} \frac{\sum_{k=r}^{w}\left(\begin{array}{l}
w \\
k
\end{array}\right) \frac{1}{c_{k}}}{\sum_{k=0}^{w}\left(\begin{array}{l}
w \\
k
\end{array}\right) \frac{1}{c_{k}}},
$$

where

$$
C_{r}=\prod_{l=1}^{r} \frac{\alpha_{l}}{1-\alpha_{l}}
$$


After obtaining a closed form expression of $B_{r}$, we can consequently find our steady-state occupancy probabilities $\pi_{k}$ by substituting for $B_{r}$ from Eq. (28) in Eq. (25). From the steady-state occupancy probabilities $\pi_{k}$, which define the steady-state probabilities of the number of customers in the system as seen by incoming customers (imbedded Markov points), we obtain the average burst loss probability, denoted by $P_{L}$, as follows:

$$
\begin{aligned}
P_{L} & =\operatorname{Pr}\{\text { Incoming DB sees } w \text { customers in the system }\} \\
& =\lim _{n \rightarrow \infty} \operatorname{Pr}\left\{q_{n}=w\right\} \\
& =\pi_{w}
\end{aligned}
$$

which characterizes the performance of the OBS core node considered.

Obviously, the results of this subsection apply directly to the FWC case where servers are fully accessible. However, they can also be used to study the NWC case by modeling each output port in the node as a $w$ independent $G I / M / 1 / 1$ queue, each having a mean arrival rate $\lambda / w$, and calculating $P_{L}$ for only one of them, which is exactly the same as the overall $P_{L}$.

Finally, it is noteworthy that the evaluated steady-state occupancy probabilities, and hence the average burst loss probability, are valid for any arrival process provided that we know the Laplace transform of the pdf of inter-arrival times. Hence, for the sake of comparison in the results section, we will use the equations of this subsection to evaluate $P_{L}$ in the FWC and NWC cases for two traffic types: LRD Pareto arrivals and SRD Poisson arrivals.

\section{B. GI/M/w/w Queue With Partial Server Accessibility}

Our next target is to analyze the system when the number of convertible wavelengths is less than $w$, which can be mathematically formulated as a $G I / M / w / w$ queue with partial server accessibility. In this case, we should modify the transition probabilities of the imbedded DTMC $\left\{q_{n}\right\}$ presented in the preceding subsection, to account for the partial server accessibility. Furthermore, the degree of restriction faced by incoming DBs while accessing servers (wavelengths on the destination output port) has to be dependent on the node conversion capability $\gamma$, where $0 \leq \gamma \leq 1$.

In order to include that behavior in the transition probabilities of $\left\{q_{n}\right\}$, we define for state $i$, where $i \in\{0,1, \ldots, w-1\}$,

$$
\begin{aligned}
\beta_{i}= & \operatorname{Pr}\{\text { incoming DB is accepted }\} \\
= & \operatorname{Pr}\{\text { incoming DB rides on a free wavelength }\} \\
& +\operatorname{Pr}\{\text { incoming DB rides on a busy wavelength }\} \\
& \times \operatorname{Pr}\{\text { busy wavelength of incoming DB is convertible }\} \\
= & \frac{w-i}{w}+\frac{i}{w} \times \gamma
\end{aligned}
$$

It is important to notice that $\beta_{i}$ is defined for all states except the last one, i.e., when $q_{n}=w$, because, in this case, it is impossible to serve the arriving DB as all servers are busy even if there are free wavelength converters.
The state transition probability diagram of the imbedded DTMC $\left\{q_{n}\right\}$ in the partial server accessibility case is the same as the one shown in Fig. 2 except for modifying the transition probabilities from the ones calculated in the previous subsection for the full server accessibility case. Clearly, according to the value of $i$, we have two cases for the transition probabilities $p_{i j}$ as follows:

a. Case $i<w$ : In this case, the new arrival is accepted with probability $\beta_{i}$ or dropped with probability $1-\beta_{i}$.

Obviously, for $j>i+1, p_{i j}=0$. On the other hand, for $j<i+1$, we have

$$
\begin{aligned}
p_{i j}= & \operatorname{Pr}\{\text { incoming DB is accepted }\} \\
& \times \operatorname{Pr}\left\{i+1-j \mathrm{DB} \text { are served during } T_{a}^{n+1}\right\} \\
& +\operatorname{Pr}\{\text { incoming DB is rejected }\} \\
& \times \operatorname{Pr}\left\{i-j \text { DBs are served during } T_{a}^{n+1}\right\} \\
= & \beta_{i} \int_{0}^{\infty}\left(\begin{array}{c}
i+1 \\
j
\end{array}\right)\left(1-e^{-\mu t}\right)^{i+1-j} e^{-j \mu t} f_{T_{a}}(t) d t \\
& +\left(1-\beta_{i}\right) \int_{0}^{\infty}\left(\begin{array}{c}
i \\
j
\end{array}\right)\left(1-e^{-\mu t}\right)^{i-j} e^{-j \mu t} f_{T_{a}}(t) d t,
\end{aligned}
$$

while for $j=i+1$, the new arrival has to be accepted for the DTMC to make the transition from state $i$ to $i+1$, and hence

$$
p_{i j}=p_{i, i+1}=\beta_{i} \int_{0}^{\infty} e^{-(i+1) \mu t} f_{T_{a}}(t) d t
$$

A simple way to check the correctness of the above transition probabilities for the case $i<w$ is by verifying that $\sum_{j} p_{i j}=1$ by plugging in $p_{i j}$ from Eqs. (32) and (33).

b. Case $i=w$ : In this case, the new arrival is dropped and $p_{i j}$ is unchanged from the full server accessibility case given by Eq. (18).

Now, we proceed exactly the same as we did for the full server accessibility case towards finding the steady-state occupancy probabilities of the imbedded DTMC. After some algebraic manipulations, the $\operatorname{pgf} \Pi(z)$ is found as

$$
\begin{aligned}
\Pi(z)= & \sum_{k=0}^{w} \pi_{k} \int_{0}^{\infty}\left(1-e^{-\mu t}+z e^{-\mu t}\right)^{k+1} f_{T_{a}}(t) d t \\
& +\gamma(1-z) \pi_{w} \int_{0}^{\infty} e^{-\mu t}\left(1-e^{-\mu t}+z e^{-\mu t}\right)^{w} f_{T_{a}}(t) d t \\
& +\frac{(1-\gamma)(1-z)}{w} \sum_{k=0}^{w} k \pi_{k} \int_{0}^{\infty} e^{-\mu t} \\
& \times\left(1-e^{-\mu t}+z e^{-\mu t}\right)^{k} f_{T_{a}}(t) d t
\end{aligned}
$$

Then, we find the binomial moments $B_{r}$ of $\Pi(z)$, defined as in Eq. (24), by applying the operator $R_{r}$ to Eq. (34), which gives the following first order difference equation:

$$
\begin{aligned}
B_{r}= & \alpha_{r}\left[B_{r}+B_{r-1}\right]-\gamma \alpha_{r}\left(\begin{array}{c}
w \\
r-1
\end{array}\right) B_{w} \\
& -\frac{(1-\gamma)}{w} \alpha_{r}\left[r B_{r}+(r-1) B_{r-1}\right],
\end{aligned}
$$


where $\alpha_{r}$ is the same as defined in Eq. (27). Next, the above difference equation can be solved recursively until $B_{r}$ is found as

$$
B_{r}=C_{r} \frac{\sum_{l=r}^{w}\left(\begin{array}{l}
w \\
l
\end{array}\right) \frac{1}{c_{l}\left(1-k_{l}\right)}}{\sum_{l=0}^{w}\left(\begin{array}{c}
w \\
l
\end{array}\right) \frac{1}{c_{l}\left(1-k_{l}\right)}},
$$

where

$$
C_{r}=\prod_{i=1}^{r} \frac{\alpha_{i}\left(1-k_{i-1}\right)}{1-\alpha_{i-1}\left(1-k_{i}\right)}
$$

and

$$
k_{i}=\frac{(1-\gamma) i}{w}
$$

Next, we find our steady-state occupancy probabilities $\pi_{k}$ from the obtained binomial moments by substituting for $B_{r}$ from Eq. (36) into Eq. (25). Finally, we obtain the average burst loss probability $P_{L}$ from the steady-state occupancy probabilities $\pi_{k}$ as

$P_{L}=\operatorname{Pr}\{$ incoming DB finds $w$ customers in system $\}$

$$
+\sum_{i=1}^{w-1}\left(\begin{array}{c}
\operatorname{Pr}\{\text { incoming DB finds } i \text { customers in system }\} \\
\times \operatorname{Pr}\{\text { incoming DB rides on busy wavelength }\} \\
\times \operatorname{Pr}\{\text { its wavelength is nonconvertible }\}
\end{array}\right)
$$

$$
=\pi_{w}+\sum_{i=1}^{w-1} \pi_{i} \times \frac{i}{w} \times(1-\gamma),
$$

which is the performance measure of the core node considered.

\section{Simulation AND NUMERICAL RESUlTS}

First, a simulation is made under MATLAB to validate our mathematical model. In this simulation, Pareto distributed arrivals, with $H$ and $\lambda$ generic, and exponentially distributed DB lengths, with $\mu$ generic, are generated. Then, assuming PWC, with $\gamma$ also left generic, the average burst loss probability $P_{L}$ is calculated as the ratio between the number of irresolvable contentions and the total number of generated arrivals. Figure 3 shows the comparison between the values of $P_{L}$ obtained from the model and simulation in the NWC, PWC $(\gamma=0.5)$ and FWC cases. In all the curves, $P_{L}$ is drawn against $\lambda$ in bursts/time unit while fixing $H$ at 0.75 and $1 / \mu$ at 50 time units. The results of both the model and the simulation prove to be in good agreement for the shown traffic range. Moreover, when the NWC, PWC and FWC cases are compared together, it is interesting to see that adding wavelength conversion is more effective for small values of $\lambda$. This is because, in light traffic scenarios, there are more free wavelengths to which a contending DB can be converted.

Next, we use our model to compare between the values of $P_{L}$ in two cases: Pareto LRD arrivals and Poisson SRD

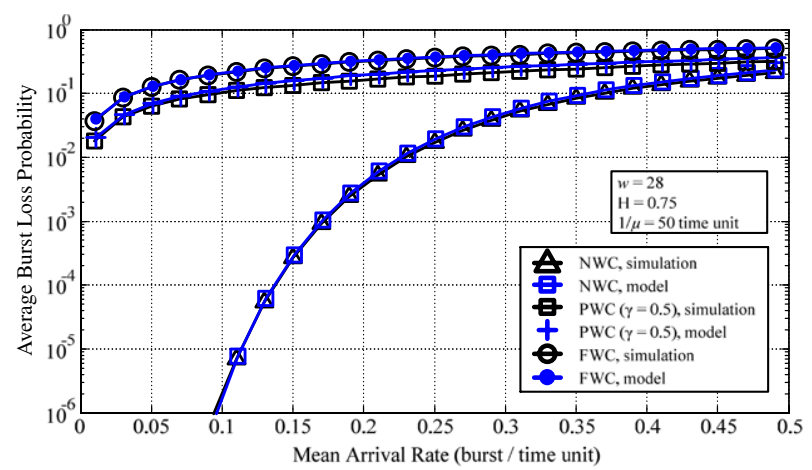

Fig. 3. (Color online) Average burst loss probability versus mean arrival rate for both the mathematical model and the simulation in the NWC, PWC and FWC cases assuming Pareto arrivals.

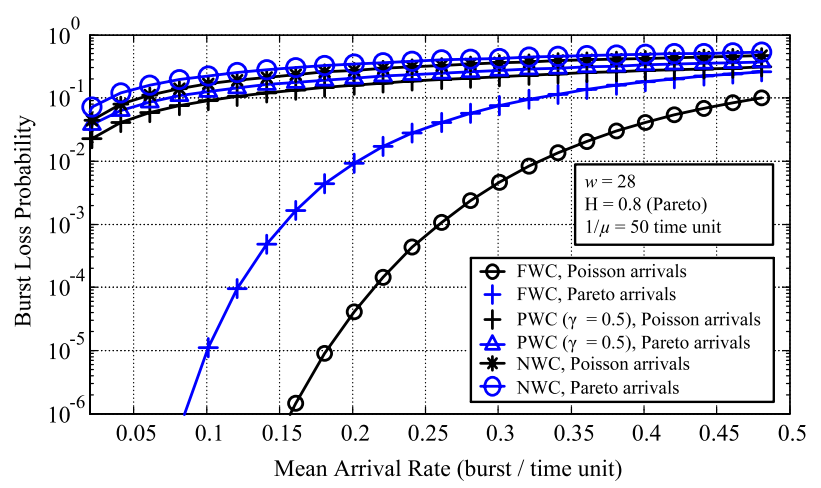

Fig. 4. (Color online) Comparison of the average burst loss probability versus the mean arrival rate for Pareto and Poisson arrivals in the NWC, PWC and FWC cases.

arrivals. For Pareto arrivals, we use Eq. (6) for the Laplace transform of the inter-arrival time distribution $F_{T_{a}}(s)$. On the other hand, for Poisson arrivals whose inter-arrivals are exponentially distributed with parameter $\lambda$, we use $F_{T_{a}}(s)=$ $\lambda /(s+\lambda)$. Also, we can alternatively study the case of Poisson arrivals by considering an $M / M / w / w$ queue with partial server accessibility from the start like our work in [6]. In Fig. 4, we plot $P_{L}$ against $\lambda$ in the NWC, PWC $(\gamma=0.5)$ and FWC cases for both Poisson arrivals and Pareto arrivals (having $H=0.8$ ) with the mean arrival rate $\lambda$ held the same for both. Thus, we conclude that mathematical models based on conventional Markovian traffic models give lower $P_{L}$ when compared to their values when the self-similarity or LRD property is considered. Moreover, the difference between the values of $P_{L}$ for SRD and LRD traffic is much clearer for lower values of $\lambda$. This is because heavy-tailed distributions like the Pareto distribution assign a higher probability for large values of inter-arrival times compared to the Poisson distribution, noting that the significance of these large inter-arrival times is clearer in the case of light traffic.

Afterwards, in Fig. 5, we employ the model to study how varying the node conversion capability affects the average loss probability for various degrees of self-similarity. Obviously, increasing the conversion capability improves the performance for all degrees of self-similarity. Furthermore, we notice that 


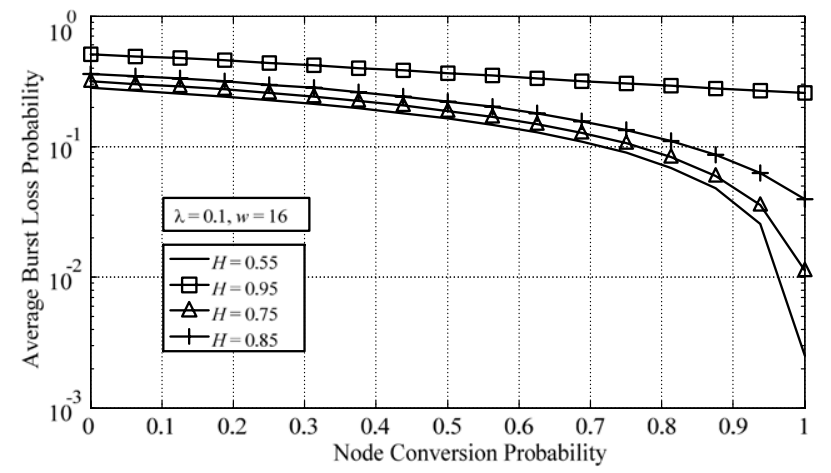

Fig. 5. Semi-log plot for average burst loss probability versus node conversion capability for Pareto arrivals at different values of the Hurst parameter.

as we go from NWC to FWC, $P_{L}$ in the case of $H=0.55$ is improved by two orders of magnitude more than the case of $H=0.95$, which interestingly means that the improvement obtained by adding more wavelength converters decreases as the traffic stream exhibits stronger LRD. This can be explained by realizing the fact that the probability of an extremely large event in an LRD process is non-negligible, i.e., extremely large inter-arrivals occur from time to time in a Pareto traffic stream. This higher degree of burstiness of arrival traffic makes it less probable for an arriving DB to find a free wavelength converter compared to smoother Poisson SRD traffic. By smoother Poisson traffic we mean that the number of arriving DBs in a time window tends to be constant as the window size increases, while for Pareto arrivals, the process preserves its randomness over very long time scales. Hence, intuitively, allocating free wavelength converters for incoming Pareto traffic is harder than for Poisson traffic.

From Fig. 5, it might appear that setting the conversion capability to its maximum value, i.e., $\gamma=1$, would seemingly improve the performance for all degrees of self-similarity of the traffic stream, although the extent of improvement is less for higher LRD traffic. However, FWC is not necessarily the perfect choice. From a system design perspective, implementing FWC in each node is highly expensive. Thus, we should try to take into account the cost of implementing such costly devices. The key idea we use is as follows: we find the overall cost of resources in each node as the cost of wavelength channels and wavelength converters, which can be formulated as

$$
\text { Overall Cost }=c_{1} w+c_{2} u
$$

where $c_{1}$ and $c_{2}$ are the cost of adding one wavelength and one wavelength converter, respectively. Also, one should be careful that the overall cost and cost per unit used above do not represent the actual prices of components; however, they are an overall measure of technological complexity, commercial availability and price. Later, we have something to say about the choice of $c_{1}$ and $c_{2}$. Turning to Eq. (40), it can be written as

$$
\text { Overall Cost } / c_{1}=w\left(1+r_{c} \gamma\right) \text {, }
$$

where the cost ratio parameter is defined as $r_{c} \stackrel{\text { def }}{=} c_{2} / c_{1}$.

In order to take the cost of adding converters into account, the idea is to trade off adding more converters by decreasing the number of wavelengths to hold the overall cost of resources constant. In that way, some degree of fairness is achieved in assessing the improvement due to adding converters. Thus, fixing the overall cost in Eq. (41), we have

$$
w\left(1+r_{c} \gamma\right)=\text { const }=w_{\mathrm{NWC}},
$$

where $w_{\text {NWC }}$ is the number of wavelengths when $\gamma=0$, which is a chosen constant as design requires.

As is seen from Eq. (42), with $w_{\mathrm{NWC}}$ and $r_{c}$ held constant, increasing $\gamma$ will decrease $w$ in such a manner that the overall cost is fixed. Obviously, $r_{c}$ is a parameter chosen according to the complexity of adding converters relative to adding wavelengths. Basically, choosing $r_{c}$ totally depends on application-specific considerations. In some cases, for example, the cost of adding channels to running systems might be almost negligible as there may be unused ports in MUX/DEMUX units (AWGs, for example) and unused transceivers at edge nodes. In such cases, adding one wavelength channel involves no additional cost and is merely done by lighting it up. In other cases, this may involve installation of extra transceivers at edge nodes. Also, adding channels sometimes requires modification of the launch power levels to ensure that amplifiers (if there are any) do not get saturated, which imposes an additional cost of re-engineering the link budget. Moreover, switching units installed in core nodes should be able to accommodate the increase in overall number of channels and this may necessitate using more advanced switching technologies, which adds to the overall cost. On the other hand, the complexity of adding wavelength converters is greatly determined by commercial availability. Most current wavelength converter products fit research applications rather than large-scale commercial applications. For example, one problem of these products is that their packaging may not be suitable for easy deployment in an installed switching node. One more issue is that all converters have to be pumped properly for the conversion mechanism to be initiated, noting that the pump lasers used impose biasing and stabilization requirements. According to the preceding discussion, $r_{c}$ totally depends on application related issues and differs from one scenario to another. However, our mathematical model and method of assessing wavelength conversion are not constrained to a specific value of $r_{c}$.

In Fig. 6, we apply the described technique to assess the effectiveness of adding wavelength conversion while accounting for its cost. Basically, the loss probability is drawn versus $\gamma$ while using Eq. (42) to determine the number of wavelengths at each $\gamma$. In the four curves at the top of Fig. 6, fixed values of $w_{\mathrm{NWC}}=16, \lambda=0.2,1 / \mu=50$ and $r_{c}=1$ are used while $H$ is varied. Clearly, the optimum value of $\gamma$ that leads to the smallest $P_{L}$, denoted by $\gamma_{\text {opt }}$, is not 1 , and hence FWC is not always the optimum choice from both performance and cost perspectives. Also, we notice that as $H$ increases, $\gamma_{\text {opt }}$ decreases, because the effectiveness of wavelength conversion is reduced significantly for higher self-similar traffic. Amazingly, for example, when $H=0.96, \gamma_{\text {opt }}=0$, which means that NWC is the optimal choice in this case compared to $\gamma_{\text {opt }} \cong 0.6$ when $H=0.55$. Finally, in the bottom two curves, we demonstrate the effect of the cost ratio parameter on $\gamma_{\mathrm{opt}}$. We use $w_{\mathrm{NWC}}=32, \gamma=0.1$, $1 / \mu=50$ and $H=0.55$ as fixed values while setting $r_{c}=1$ and 2 . 


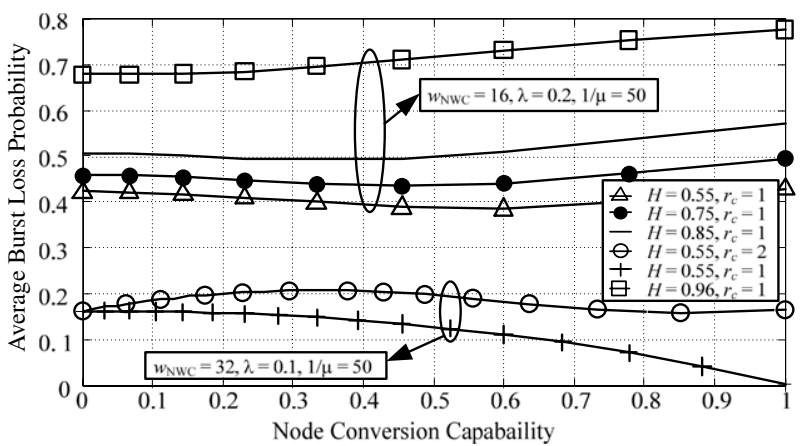

Fig. 6. Average burst loss probability versus node conversion capability, taking the overall cost of resources into consideration, for Pareto arrivals at different values of the Hurst parameter and the cost ratio parameter.

Noticeably, $\gamma_{\mathrm{opt}}=1$ for $r_{c}=1$ and decreases to $\gamma_{\mathrm{opt}} \cong 0.85$ when $r_{c}=2$. This is justified by the fact that, as $r_{c}$ increases, $\gamma_{\text {opt }}$ decreases, as converters become more expensive relative to wavelengths.

\section{CONCLUSION}

From the aforementioned results and observations, we can draw the following conclusions:

- The results of our mathematical model prove to be in good agreement with simulation results, which justifies the model usage for Pareto arrivals in the NWC, PWC and FWC cases.

- Wavelength conversion generally improves the average burst loss probability; however, it becomes more effective in lighter traffic scenarios for either Pareto or Poisson arrivals.

- Mathematical models based on conventional memory-less traffic models anticipate over-optimistic performance when compared to those resulting from more complicated models based on traffic models that capture the self-similar or LRD nature of truly bursty Internet traffic. Furthermore, the results of these conventional Markovian models deviate further from their peer LRD models as incoming traffic becomes lighter.

- As the traffic becomes more self-similar, the improvement obtained by adding wavelength converters to the node resources decreases significantly.

- Taking the cost of adding wavelength converters into consideration, an optimum degree of conversion capability proves to exist. This optimum degree of conversion, or equivalently optimum number of converters, tends to decrease as the traffic becomes more self-similar, to the extent that NWC accompanied by the use of more wavelength channels may be the optimum solution.

\section{FUTURE WORK}

In the presented work, several approximations have been made with respect to the real system. First, we assumed that the burst length follows an exponential distribution, while it actually depends on the system under test, the traffic at the ingress node and the burst assembly algorithm used. Next, we assumed a perfect control plane where the CPs do not suffer any kind of blocking. Consequently, the calculated burst loss probability can be considered as the burst loss probability given that the CP arrival succeeded. Such assumptions can be relaxed in order to gradually accommodate the model to the real system.

Furthermore, the performance of an OBS network is affected by many other factors such as different offset times, the scheduling algorithm used and the burst assembly algorithm adopted. However, studying the performance of a single OBS core node still gives insight into how the node will perform with the parameters assumed in a networking scenario. Thus, the presented study can be extended to build a full network model, whose building block would be our model presented in this work, to calculate link blocking probabilities.

Finally, building a network simulation model would represent a major step in understanding how the model reflects the overall performance and is one of our future research goals.

\section{REFERENCES}

[1] M. Yoo and C. Qiao, "Just-enough-time (JET): A high speed protocol for bursty traffic in optical networks," in Proc. IEEE / LEOS Summer Topical Meetings Dig. for Conf. Technologies Global Information Infrastructure, Aug. 11-15, 1997, pp. 26-27.

[2] C. Qiao and M. Yoo, "Optical burst switching (OBS)-A new paradigm for an optical Internet," J. High Speed Netw., vol. 8, no. 1, pp. 69-84, Jan. 1999.

[3] T. Battestilli and H. Perros, "An introduction to optical burst switching," IEEE Commun. Mag., vol. 41, no. 8, pp. S10-S15, Aug. 2003

[4] X. Yu, J. Li, X. Cao, Y. Chen, and C. Qiao, "Traffic statistics and performance evaluation in optical burst switched networks," J. Lightwave Technol., vol. 22, no. 12, pp. 2722-2738, Dec. 2004.

[5] I. Baldine, G. N. Rouskas, H. G. Perros, and D. Stevenson, "JumpStart: A just-in-time signaling architecture for WDM burst-switched networks," IEEE Commun. Mag., vol. 40, no. 2, pp. 82-89, Feb. 2002.

[6] G. Wu, T. Zhang, J. Chen, X. Li, and C. Qiao, "An index-based parallel scheduler for optical burst switching networks," J. Lightwave Technol., vol. 29, no. 18, pp. 2766-2773, Sept. 2011.

[7] L. H. Bonani, "Performance evaluation of optical burst switched mesh networks with Localized Functional Resources," in Proc. Int. Conf. on Transparent Optical Networks (ICTON), Coventry, England, July 2-5, 2012, pp. 1-4.

[8] H. Ichikawa and K. Kamakura, "Dimensioning an scheduler buffer in OBS networks using forward resource reservation," in Proc. Int. Conf. on Computing, Networking and Communications (ICNC), Maui, Hawaii, Jan. 30-Feb. 2, 2012, pp. 282-286.

[9] A. I. Abd El-Rahman, S. I. Rabia, and H. M. H. Shalaby, "MAC-layer performance enhancement using control packet buffering in optical burst switched networks," J. Lightwave Technol., vol. LT-30, no. 11, pp. 1578-1586, June 2012.

[10] N. Akar, E. Karasan, and K. Dogan, "Wavelength converter sharing in asynchronous optical packet/burst switching: An exact blocking analysis for Markovian arrivals," IEEE J. Sel. Areas Commun., vol. 24, no. 12, pp. 69-80, Dec. 2006. 
[11] M. Y. S. Sowailem, M. H. S. Morsy, and H. M. H. Shalaby, "Employing code domain for contention resolution in optical burst switched networks with detailed performance analysis," J. Lightwave Technol., vol. LT-27, pp. 5284-5294, Dec. 2009.

[12] M. H. S. Morsy, M. Y. S. Sowailem, and H. M. H. Shalaby, "Performance analysis of a core node equipped with wavelength converter pool in an Optical Burst Switched network," in Proc. 2010 IEEE 17th Int. Conf. on Telecommun. (ICT), Apr. 4-7, 2010, pp. 516-522.

[13] Z. Rosberg, A. Zalesky, H. L. Vu, and M. Zukerman, "Analysis of OBS networks with limited wavelength conversion," IEEE / ACM Trans. Netw., vol. 14, no. 5, pp. 1118-1127, Oct. 2006.

[14] D. Morato, M. Izal, J. Aracil, E. Magana, and J. Miqueleiz, "Blocking time analysis of OBS routers with arbitrary burst size distribution," in Proc. Globecom 2003, Dec. 1-5, 2003, vol. 5, pp. 2488-2492.

[15] T. Battestilli and H. G. Perros, "A performance study of an optical burst switched network with dynamic simultaneous link possession," J. Comput. Netw., vol. 50, no. 2, pp. 219-236, Feb. 2006.

[16] L. Battestilli, H. Perros, and S. Chukova, "Burst lost probabilities in a queuing network with simultaneous resource possession: A single-node decomposition approach," IET Commun., vol. 3, no. 3, pp. 441-453, Mar. 2009.

[17] D. Tafani, C. McArdle, and L. P. Barry, "A two-moment performance analysis of optical burst switched networks with shared fibre delay lines in a feedback configuration," J. Opt. Switching Netw., vol. 9, no. 5, pp. 323-335, Nov. 2012.

[18] W. Leland, M. Taqqu, W. Willinger, and D. Wilson, "On the self-similar nature of Ethernet traffic (extended version)," IEEE / ACM Trans. Netw., vol. 2, no. 1, pp. 1-15, Feb. 1994.

[19] W. Leland and D. Wilson, "High time-resolution measurement and analysis of LAN traffic: Implications for LAN interconnection," in Proc. 10th Annu. Joint Conf. of the IEEE Computers and Communications Societies, Networking in the 90s (INFOCOM), Apr. 7-11, 1991, vol. 3, pp. 1360-1366.

[20] M. H. S. Morsy, M. Y. S. Sowailem, and H. M. H. Shalaby, "Upper and lower bounds of burst loss probability for a core node in an Optical Burst Switched network with Pareto distributed arrivals," in Proc. 2010 IEEE 17th Int. Conf. on Telecommun. (ICT), Apr. 4-7, 2010, pp. 523-527.

[21] Y. Chen, H. Wu, D. Xu, and C. Qiao, "Performance analysis of optical burst switched node with deflection routing," in Proc. IEEE Int. Conf. on Commun. (ICC), May 2003, vol. 2, pp. 1355-1359.

[22] M. Yoo, C. Qiao, and S. Dixit, "QoS performance of optical burst switching in IP-over-WDM networks," IEEE J. Sel. Areas Commun., vol. 18, no. 10, pp. 2062-2071, Oct. 2000.

[23] H. L. Vu and M. Zukerman, "Blocking probability for priority classes in optical burst switching networks," IEEE Commun. Lett., vol. 6, no. 5, pp. 214-216, May 2002.

[24] S. Nadarajah and S. Kotz, "On the Laplace transform of the Pareto distribution," IEEE Commun. Lett., vol. 10, no. 9, pp. 682-682, Sept. 2006.

[25] L. Kleinrock, Queueing Systems Volume 1: Theory. Wiley, New York, 1975

[26] L. Takacs, Introduction to the Theory of Queues. Oxford University Press, New York, 1961.

Mohamed H. Morsy-Osman was born in Cairo, Egypt, in 1985. He received his B.S. and M.S. degrees from Alexandria University, Alexandria, Egypt, in 2006 and 2009, respectively. He is currently working towards a Ph.D degree at the Department of Electrical and
Computer Engineering, McGill University, Montreal, Canada. In 2010, he joined the Department of Electrical and Computer Engineering, McGill University, as a Research Assistant for two years until now. His current research interests include digital signal processing for high speed optical transport networks, optical burst switched networks (OBSNs), optical code division multiple access (OCDMA) networks, media access control (MAC) protocols in optical networks, and wireless communications.

Mohammed Y. S. Sowailem was born in Yanbu, Saudi Arabia, in 1984. He received his B.S. degree and the M.S. degree in electrical engineering from Alexandria University, Alexandria, Egypt, in 2006 and 2009, respectively. He is now working towards his Ph.D. degree at the Electrical and Computer Engineering Department, McGill University, Montreal, QC, Canada. In 2006, he joined the Basic and Applied Science Department, Arab Academy of Science and Technology, as a part time Teaching Assistant for one year. Then, he joined SySDSoft, Inc. as a DSP Engineer from August 2007 to November 2009. From 2010 until now, he has been at the Electrical and Computer Engineering Department, McGill University, as a Research Assistant. His current research interests include optical burst switched networks in data centers, optical code division multiple access (OCDMA) networks, media access control (MAC) protocols in optical networks, computer networks, and wireless communications.

Ahmed I. Abd El-Rahman (S'11) was born in Alexandria, Egypt, in 1979. He received his B.S. and M. S. degrees from Alexandria University, Alexandria, Egypt, in 2002 and 2012. In 2002, he joined Fardos, Alexandria, Egypt, for investment and construction as a Project Assistant Manager. His current research interests include optical burst switched networks (OBSNs), optical orthogonal frequency division multiplexing (OOFDM), optical CDMA (OCDMA) and wireless systems.

Hossam M. H. Shalaby (S'83-M'91-SM'99) was born in Giza, Egypt, in 1961. He received his B.S. and M.S. degrees from Alexandria University, Alexandria, Egypt, in 1983 and 1986, respectively, and his Ph.D. degree from the University of Maryland at College Park in 1991, all in electrical engineering.

In 1991, he joined the Electrical Engineering Department, Alexandria University, and was promoted to Professor in 2001. Currently he is on leave from Alexandria University, where he is the chair of the Department of Electronics and Communications Engineering, School of Electronics, Communications, and Computer Engineering, Egypt-Japan University of Science and Technology (E-JUST), New Borg El Arab City, Alexandria, Egypt. From December 2000 to 2004, he was an Adjunct Professor with the Faculty of Sciences and Engineering, Department of Electrical and Information Engineering, Laval University, Quebec, QC, Canada. From September 1996 to February 2001, he was on leave from Alexandria University. From September 1996 to January 1998, he was with the Electrical and Computer Engineering Department, International Islamic University Malaysia, and from February 1998 to February 2001, he was with the School of Electrical and Electronic Engineering, Nanyang Technological University, Singapore. His research interests include optical communications, optical CDMA, optical burst switching, OFDM technology and information theory.

Dr. Shalaby has served as a student branch counselor at Alexandria University, IEEE Alexandria and North Delta Subsection, from 2002 to 2006, and served as a chairman of the student activities committee of IEEE Alexandria Subsection from 1995 to 1996. He received an SRC fellowship from 1987 to 1991 (Systems Research Center, Maryland), State Excellence Award in Engineering Sciences in 2007 (Academy of Scientific Research and Technology, Egypt), Shoman Prize for Young Arab Researchers in 2002 from The Abdul Hameed Shoman Foundation, Amman, Jordan, the State Incentive Award in Engineering Sciences in both 1995 and 2001 from the Academy of Scientific Research and Technology, Egypt, the University Excellence Award in 2009 from Alexandria University and the University Incentive Award in 1996 from Alexandria University. 